\title{
Editorial
}

\author{
Lorenza S. Colzato $^{1,2}$
}

Published online: 28 June 2016

(C) Springer International Publishing 2016

Starting up a new journal is an outstanding possibility to consider the directions into which the targeted field is currently heading. The journal will put research on cognitive enhancement into a broader perspective and in closer connection with (a) other scientific areas and disciplines and (b) the social and infrastructural changes of modern society in order to truly achieve an integrative science of cognitive enhancement. Cognitive enhancement is not just one more psychological intervention but it touches important societal and ethical issues.

\section{The Time is Now}

Higher, faster, more this is what my trainer used to tell me before every fencing competition in order to get the best performance out of me. In sport, just like in science, I was more motivated too reach my personal best than outcompete others. This is for me the essence of cognitive enhancement: the use of any (legitimate) means (e.g., video game, brain stimulation, neurofeedback, physical exercise, or food supplements) to reach one's personal best.

Why have a journal about cognitive enhancement now? Why has this topic become so relevant in the past few years?

Economically, the interest in cognitive enhancement is mainly driven by the increasing costs of the welfare system,

Lorenza S. Colzato

colzato@fsw.leidenuniv.nl

1 Institute for Psychological Research and Leiden Institute for Brain and Cognition, Leiden University, Leiden, The Netherlands

2 Institute for Psychological Research, Cognitive Psychology Unit, Leiden University, Wassenaarseweg 52, 2333, AK

Leiden, The Netherlands especially with regard to the increasing age of citizens in Western societies. For instance, cognitive enhancement can help to delay cognitive decline in the elderly, which would extend the time people can live autonomously and, thus, reduce the welfare costs for the time thereafter. Along the same lines, training children could speed up the education of healthy individuals and reduce the risk of behavioral deviance and pathology, again with considerable savings for welfare and education systems. But, there is also a more ideological reason for the increased interest in cognitive enhancement. Both Eastern and Western societies are continuously driven towards more individualism, which emphasizes the existence and often also the importance of individual differences over commonalities and collectivistic values. These tendencies go hand in hand with ideological developments in public opinion and within political parties, which in many countries have gravitated towards more neoliberal, individualism-heavy positions over the last 15 years or so. Among other things, this has involved a rather systematic deconstruction of the welfare system and established the view of the individual as an architect of his or her own life. Research on cognitive enhancement has benefited from both aspects of this trend. The economic problems of the welfare system have boosted the interest in procedures and activities that make welfare societally more affordable, and the ideological turn towards individualism provides a natural breeding ground for the public interest in procedures and activities that help to express and to further develop individual needs and interests.

\section{Funding Agencies Promote Cognitive Enhancement}

The widespread use of smart phones has led to a real explosion of "apps" to enhance cognitive functioning, ranging from simple alerts reminding the elderly to take her/his pill to 
theoretically guided programs to systematically enhance specific cognitive functions, such as in the case of the app PEAK. Industry and funding agencies have taken notice of the many opportunities these techniques can open, and the current European Research Area (ERA in Horizon 2020) has various calls to promote gamification. Along the same line, president Obama declared already in 2011 the importance of investing money in educational technology and realized the enhancing potential of gaming. Indeed, as quoted from the White House initiative "Games that Can Change the World": "Games for impact are designed to be at once entertaining and engaging, and also something more: educational, enlightening, and perhaps even designed to motivate action." Clearly, this is likely to strengthen this trend further in the near future, but I think that the full potential of gamification is not always sufficiently appreciated. Turning psychological experiments and training procedures into apps is certainly handy for both researchers and users, especially as it allows to integrate cognitive enhancing programs better with real-life circumstances. Another example to demonstrate how ERA is focusing on cognitive enhancement is the Joint Programming Initiative "A healthy diet for a healthy life" which is funding research on how nutrients can prevent (social) cognitive decline in normal aging. This initiative is particularly emblematic in showing how economic problems of the welfare system have boosted the interest in procedures and activities that make welfare societally more affordable.

\section{Need for Theory}

There are not many where Kurt Lewin's claim that "nothing is as practical as a good theory" does not apply, but hardly any to which it applies more than the area of cognitive enhancement. Whereas in the past the field of cognitive enhancement used mainly effect-driven approaches (that seek to demonstrate that an enhancing intervention can have an effect without explaining how it modulates the targeted function and why some people benefit more than others), this journal pursues a mechanistically oriented, theory-driven approach that tries to understand and explain individual differences to a degree that allows a comprehensive understanding of how a particular intervention modulates (social) cognition.

\section{Scientific Formats and Support of Open Science Framework}

Beyond the classical formats, such as research, opinion, hypothesis, and theory articles, we are considering a number of new formats that are likely to propagate innovation. One is the Mini Review, in times of informational overload there is an increasing need for papers that pinpoint the state-of-the-art in a particular area. Such articles should not always consider the complete history of a particular topic or effect if they only capture the state of the art and the major theoretical implications: the shorter the better. Another one is Support of the Null Hypothesis. If supported by appropriate statistical evidence (such as Bayesian inference) and by strong theoretical predictions, this journal welcomes research articles that do not reject the null hypothesis. Finally, this journal supports the mission of the center for open science to increase openness, integrity, and reproducibility of scientific research. The (raw) data of the research articles that will be reported in this journal are expected to be stored in the open science framework.

\section{Concluding remarks}

The editorial board and myself are enthusiastic about making the journal attractive for top scientists in the area of cognitive enhancement and to readers interested in this emerging topic. At present, authors experience increasing difficulties to get their contributions published, because the traditional journals are not yet ready for this market. For standard cognitive journals, the topic is too applied, and for many apply journals it is too broad, e.g., journals on meditation capture the meditation studies but do not accept studies on other, theoretically comparable enhancement techniques. Hence, this journal has the ambition to lead as a "whole" this new emerging field.

Last, I will be grateful to our readership and the authors who consider the journal suitable as an outlet of their work, and I would appreciate any suggestions as to how our services can be further improved. 